\title{
Two resource distribution strategies for dynamic mitigation of influenza pandemics
}

This article was published in the following Dove Press journal:

Journal of Multidisciplinary Healthcare

5 July 2010

Number of times this article has been viewed

\author{
Andrés Uribe-Sánchez \\ Alex Savachkin \\ Department of Industrial and \\ Management Systems Engineering \\ University of South Florida, Tampa, \\ FL 33620, USA
}

\begin{abstract}
As recently pointed out by the Institute of Medicine, the existing pandemic containment and mitigation models lack the dynamic decision support capabilities. We present two simulation-based optimization models for developing dynamic predictive resource distribution strategies for cross-regional pandemic outbreaks. In both models, the underlying simulation mimics the disease and population dynamics of the affected regions. The quantitybased optimization model generates a progressive allocation of limited quantities of mitigation resources, including vaccines, antiviral, administration capacities, and social distancing enforcement resources. The budget-based optimization model strives instead allocating a total resource budget. Both models seek to minimize the impact of ongoing outbreaks and the expected impact of potential outbreaks. The models incorporate measures of morbidity, mortality, and social distancing, translated into the societal and economic costs of lost productivity and medical expenses. The models were calibrated using historic pandemic data and implemented on a sample outbreak in Florida, with over four million inhabitants. The quantity-based model was found to be inferior to the budget-based model, which was advantageous in its ability to balance the varying relative cost and effectiveness of individual resources. The models are intended to assist public health policy makers in developing effective distribution policies for mitigation of influenza pandemics.
\end{abstract}

Keywords: pandemic, mitigation, distribution, dynamic, resources, budget

\section{Introduction and motivation}

Influenza pandemics have historically ensued enormous societal calamities amplified by staggering economic forfeitures. In the U.S. alone, the Spanish flu (1918, serotype H1N1), the Asian flu (1957, H2N2), and the Hong Kong flu (1968, H3N2) resulted in the death toll of more than 500,000, 70,000 and 34,000 cases, respectively. ${ }^{1}$ More recently, a series of scattered outbreaks of the avian-to-human transmittable H5N1 virus has been mapping its way through Asia, the Pacific region, Africa, the Near East, and Europe. ${ }^{2}$ As of March 2010, the World Health Organization (WHO) has reported 287 deaths in 486 cases, worldwide. ${ }^{3}$ At the same time, in Spring 2009, a milder human-to-human transmissible $\mathrm{H} 1 \mathrm{~N} 1$ virus subtype resurfaced and propagated to an ongoing global outbreak; as of March 2010, 213 countries have been affected with a total number of infections and mortalities of 419,289 and 16,455, respectively. ${ }^{4}$ Today, most experts have an ominous expectation that the next pandemic will be triggered by an emerging highly pathogenic virus, to which there is little or no pre-existing immunity in humans. ${ }^{5}$

The ability to contain and mitigate influenza pandemics depends on available emergency response infrastructure and resources, and at present, challenges abound.
Correspondence: Alex Savachkin

E Fowler Avenue, ENBI I8,Tampa,

FL 33620, USA

Tel + I-8I 3-974-5577

Fax + I-8I3-974-5953

Email alexs@usf.edu
Journal of Multidisciplinary Healthcare 2010:3 65-77

(c) 2010 Uribe-Sánchez and Savachkin, publisher and licensee Dove Medical Press Ltd.This is an Open

Access article which permits unrestricted noncommercial use, provided the original work is properly cited. 
Prediction of the exact emerging virus subtype remains a difficult task, and once it is identified, a surge production of sufficient vaccine quantities can take from six to nine months. ${ }^{6,7}$ Even if the emerged subtype has a known epidemiology, the existing stockpiles would be limited due to high production and inventory costs. ${ }^{8,9}$ Also will be significantly constrained the supply of antiviral, immunizers and other healthcare providers, hospital beds and supplies, and logistics. Thereby, pandemic mitigation has to be done amidst a limited knowledge of disease and population dynamics, constrained infrastructure, shortage of effective clinical treatments, and to-be-proven resource allocation policies. This challenge, faced during the recent H1N1 outbreak, has been acknowledged by $\mathrm{WHO}^{9}$ and echoed by the Health and Human Services (HHS) and the Center for Disease Control and Prevention (CDC). ${ }^{10,11}$

The existing literature on pandemic influenza (PI) modeling aims to address various complex aspects of the pandemic evolution process including: (i) underlying spatio-temporal structure, (ii) contact dynamics and disease transmission, (iii) disease progression, and (iv) development of mitigation strategies. A comprehensive decision-aid model for containment and mitigation has to invariably consider all of the above aspects: it must incorporate the mechanism of disease progression, from initial infection, to the asymptomatic phase, manifestation of symptoms, and a final health outcome; ${ }^{12-14}$ it must also consider the population dynamics, including individual susceptibility ${ }^{15,16}$ and transmissibility, ${ }^{12,17-19}$ as well as the behavioral factors that affect infection generation and disease progression; ${ }^{20-23}$ finally, it must incorporate the impact of pharmaceutical and non-pharmaceutical measures, including vaccination, antiviral therapy, social distancing and travel restrictions, and use of low-cost measures, such as face masks and hand washing. ${ }^{24-27}$

In recent years, the models for PI containment and mitigation have focused on integrating therapeutical and nontherapeutical measures in search for synergistic strategies, aimed at better resource utilization. Most of these approaches implement a form of social distancing to reduce infection exposure, followed by application of pharmaceutical means. A number of significant contributions has been made in this challenging area. ${ }^{1,25,28-32}$ One of the most notable among the recent efforts is a 2006-07 initiative by the Models of Infectious Disease Agent Study (MIDAS) ${ }^{33}$ which examined independent models of large-scale PI spread for rural areas of Asia, ${ }^{34,35}$ U.S. and U.K., ${ }^{36,37}$ and the city of Chicago. ${ }^{38}$ MIDAS cross-validated the models by simulating the city of Chicago, with $8.6 \mathrm{M}$ inhabitants, and implementing targeted layered containment. ${ }^{39,40}$ The research findings of MIDAS and other institutions ${ }^{14,25}$ were used in a recent "Modeling Community Containment for Pandemic Influenza" report by the Institute of Medicine (IOM), to formulate a set of recommendations for mitigating PI at a local level. ${ }^{41}$ These recommendations were used in a pandemic preparedness guidance developed jointly by CDC, HHS, and other federal agencies. ${ }^{42}$

At the same time, the IOM report points out several limitations of the MIDAS models, observing that "because of the significant constraints placed on the models" being considered by policy makers, "the scope of the models should be expanded." The IOM recommends "to adapt or develop decision-aid models that can ... provide real-time feedback during an epidemic." The report also emphasizes that "future modeling efforts should incorporate broader outcome measures ... to include the costs and benefits of intervention strategies". ${ }^{41}$ It can indeed be observed that practically all existing approaches focus on assessment of apriori defined strategies; virtually none of the synergistic decision models are capable of "learning", ie, adapting to changes in the pandemic course, yet predicting them, to generate a dynamic optimal strategy. Such a strategy is advantageous in its ability to be state-dependent, ie, as being formed dynamically as the pandemic spreads, by selecting the optimal combination of available mitigation options at each decision epoch, based on the present pandemic state.

In an attempt to address the IOM recommendations, we present two novel simulation-based optimization models for developing dynamic predictive resource distribution strategies for a network of regional pandemic outbreaks. In both models, the underlying simulation mimics disease and population dynamics of the affected regions (Sections 2.1 and 2.2). The quantity-based optimization model (Section 2.3) generates a progressive allocation of limited quantities of mitigation resources, including stockpiles of vaccines and antiviral, healthcare capacities for vaccination and antiviral treatment, and social distancing enforcement resources. The budget-based optimization model (Section 2.3.1) allocates a total resource budget, as opposed to a separate allocation of individual resources, which vary in their relative cost and effectiveness. Both models seek to dynamically minimize the impact of ongoing outbreaks and the expected impact of potential outbreaks, spreading from the ongoing regions. The optimality criterion incorporates measures of morbidity, mortality, and social distancing, translated into the societal and economic costs of lost productivity and medical expenses. 
The models were calibrated using historic pandemic data and implemented on a sample cross-regional outbreak in Florida, U.S., with over four million inhabitants (Section 3). We present a sensitivity analysis for estimating the marginal impact of changes in the total budget and quantity availability (Section 3.4), and show that the quantity-based approach is inferior to the budget-based strategy, which takes advantage of its ability to balance the relative cost and effectiveness of individual resources.

Compared to our earlier work, ${ }^{43}$ this paper presents the following main advances: (i) the original single-region simulation ${ }^{43}$ has been expanded to serve as the basis for the crossregional simulation model presented in this paper; (ii) we now propose two novel dynamic optimization models, embedded in the cross-regional simulation, to generate resource distribution strategies; as such, the decision support power of our model has substantially increased; (iii) the calibration methodology now incorporates both the basic reproduction number and the infection attack rate; inclusion of the latter adds to a more accurate assessment of the pandemic severity over the entirety of the pandemic period, and (iv) our model now incorporates certain socio-behavioral features, such as the target population compliance.

\section{Simulation-based optimization framework}

Our simulation-based optimization framework generates progressive resource allocations over a network of regional outbreaks. Resources include stockpiles and capacities for vaccine and antiviral administration, and social distancing enforcement resources, among others. The framework subsumes a crossregional simulation model, a set of single-region simulation models, and an overarching optimization control.

The regions inside the network are classified as unaffected, ongoing outbreak or contained. The cross-regional model connects the regions by air and land travel. The single-region model emulates the (hourly) population and disease dynamics of each ongoing region, affected by mitigation measures. The pandemic spreads from ongoing to unaffected regions by (a)symptomatic travelers passing through border control. At every new regional outbreak epoch, the optimization model allocates available resources to the new outbreak region (actual allocation) and unaffected regions (virtual allocation). Daily statistics are collected for each region, including the number of infected, deceased, and quarantined cases, for different age groups. As a regional outbreak is contained, its societal and economic costs are estimated.

\section{Cross-regional simulation model}

The model initializes by creating mixing groups and population dynamics for each region (see Section 2.1). A pandemic is triggered by injecting one infectious case into a randomly chosen region. The resulting regional contact dynamics and disease propagation are presented in Section 2.2. As the symptomatic cases start seeking medical assistance, the new regional outbreak is detected, and a resource allocation is generated (see Section 2.3) and passed over to the single-region model.

The outbreak spreads to unaffected regions as infectious travelers pass with some probability through border control (the probability varies with the degree of symptom manifestation). Travelers are assumed to act independently. Each traveler is assumed to seed a regional outbreak in her destination with an equal, time-homogeneous probability $\omega$ for the entirety of her infection period. For each unaffected region, the outbreak probability at time $t$ is calculated using the binomial probability law, as follows

$$
P_{t}=1-(1-\omega)^{n_{t}}
$$

where $n_{t}$ denotes the number of infectious travelers in the region at time $t$. Based on the outbreak probability values, the model determines which of the regions have become new outbreaks (in the testbed implementation, the values of $P_{t}$ were computed daily). The model also determines if an ongoing outbreak has been contained, based on a certain threshold of the daily infection rate. The cross-regional simulation stops when all outbreaks have been contained.

\section{Single-region simulation model}

The single-region simulation model mimics population and disease dynamics within the affected region.

A schematic of the model is shown in Figure 1. The model subsumes the following main components: (i) population dynamics (schedules), (ii) contact and infection process,

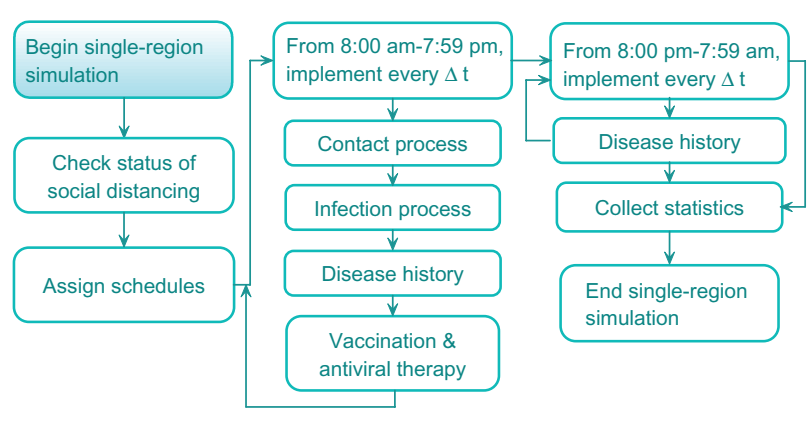

Figure I Schematic of single-region simulation model. 
(iii) disease natural history, and (iv) mitigation prevention and intervention, including social distancing, vaccination, and antiviral application. The model collects detailed regional statistics, including numbers of infected, recovered, deceased, and quarantined cases, for different age groups. For a contained outbreak, its societal and economic costs are calculated. The societal cost includes the cost of lost lifetime productivity of the deceased; the economic cost includes the cost of medical expenses of the recovered and deceased and the cost of lost productivity of the quarantined (see Section 3.3; Meltzer 1999). ${ }^{44}$ The single-region simulation extends our original model. ${ }^{43}$

\section{Population dynamics (schedules)}

We view a region as a set of population centers formed by mixing groups (eg, households, offices, facilities, universities, dormitories, schools, shopping centers, etc.). Each individual is assigned a set of attributes (eg, age, gender, parenthood, workplace, infection susceptibility, probability of travel, etc). Each person is also assigned $\Delta t$ time-discrete (eg, $\Delta t=1$ hour) weekday and weekend schedules, which depend on: (i) person's age and parenthood, (ii) disease status, (iii) travel status, (iv) social distancing decrees in place and person's compliance. As their schedules advance, inhabitants circulate throughout the mixing groups. It is assumed that at any point of time, an individual belongs to one of the exclusive compartments of susceptible, infected, recovered or deceased.

\section{Contact and infection processes}

Infection transmission occurs during contact events between susceptible and infectious cases in the mixing groups. At the beginning of every $\Delta t$ period (eg, one hour), for each mixing group $g$, the simulation tracks the total number of infectious cases, $n_{g}$, present in the group. Each infectious case generates $r_{g}$ per $\Delta t$ unit of time new contacts, ${ }^{37}$ chosen randomly (uniformly) from the susceptible. We make the following assumptions about the contact process: (i) during $\Delta t$ period, a susceptible may come into contact with at most one infectious case and (ii) each contact exposure lasts $\Delta t$ units of time. Once a susceptible has started a contact exposure at time $t$, she will develop infection at time $t+\Delta t$ with a certain probability that is calculated as shown below.

Let $L_{i}(t)$ be a nonnegative continuous random variable that represents the duration of contact exposure, starting at time $t$, required for contact $i$ to become infected. We assume that $L_{i}(t)$ is distributed exponentially with mean $1 / \lambda_{i}(t)$, where $\lambda_{i}(t)$ represents the instantaneous force of infection applied to contact $i$ at time $t .^{45-47}$ The probability that susceptible $i$, whose contact exposure has started at time $t$, will develop infection at time $t+\Delta t$ is then given as

$$
P\left\{L_{i}(t) \leq \Delta t\right\}=1-e^{-\lambda_{i}(t) \Delta t}
$$

\section{Disease natural history}

A schematic of the disease natural history is shown in Figure 2. During the incubation phase, the individual stays asymptomatic. At the end of the latency phase, she becomes infectious and enters the infectious phase..$^{35,37,39}$ She becomes symptomatic at the end of incubation. At the end of the infectious phase, she enters the final disease stage which culminates in her recovery or death.

Mortality for influenza is a complex process affected by many factors and variables, most of which have limited accurate data support from past pandemics. Furthermore, the time of death can be weeks following the disease episode (often attributable to pneumonia related complications). ${ }^{48}$ Because of the uncertainty underlying the mortality process, we thus adopted a simplified, age-based form of the mortality probability of infected $i$, as follows

$$
m_{i}=\mu_{i}-\tau \rho_{i}
$$

where $\mu_{i}$ is the age-dependent base mortality probability of infected $i, \rho_{i}$ is her status of antiviral therapy ( 0 or 1$)$, and $\tau$ is the antiviral efficacy measured as the decrease in the base probability. ${ }^{35}$ We assume that a recovered case develops immunity but continues circulating in the mixing groups.

\section{Mitigation strategies}

Mitigation include pharmaceutical and non-pharmaceutical options. It is initiated upon detection of a critical number of confirmed infected cases, ${ }^{49}$ which triggers resource allocation and deployment. We assume a certain delay for deployment of field responders.

Pharmaceutical mitigation consists of vaccination and antiviral application. Vaccination is targeted at individuals at risk to reduce their infection susceptibility. We assume that a certain fraction of the risk group does not comply

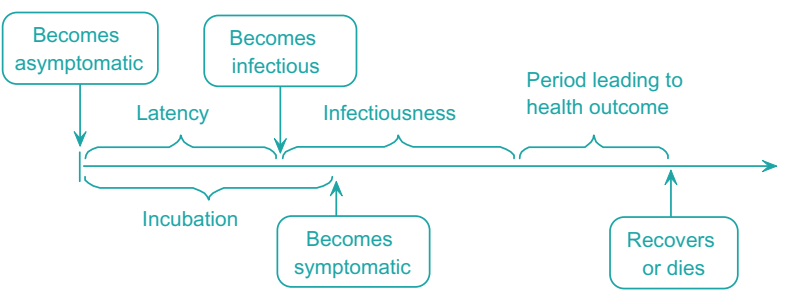

Figure 2 Schematic of disease natural history model. 
with vaccination. The vaccine takes a certain period to become effective (typically, between 10 and 14 days). ${ }^{50}$ Vaccination is constrained by the available stockpile and administration capacity measured in terms of the number of immunizer-hours.

We assume that as some symptomatic cases seek medical assistance, ${ }^{51,52} \mathrm{a}$ fraction at risk of them receives an antiviral. Antiviral treatment is subject to availability of the antiviral stockpile and administration capacity, measured in terms of the number of certified providers.

Both vaccination and antiviral application are affected by social behavioral factors, including conformance of the target population, degree of risk perception, and compliance of healthcare personnel..$^{53-55}$ The conformance level of the population at risk can be affected, among other factors, by the demographics and income level ${ }^{56-60}$ as well as the quality of public information. ${ }^{61}$ The degree of risk perception can be influenced by negative experiences developed during pharmaceutical campaigns of the previous outbreaks, ${ }^{62,63}$ as well as by public fear and rumors. ${ }^{64,65}$

Non-pharmaceutical mitigation includes social distancing and travel restrictions. We adopted a CDC guidance, ${ }^{42}$ which establishes five categories of pandemic severity and recommends quarantine and closure options according to the category. The categories are determined based on the value of the case fatality ratio (CFR), the proportion of fatalities in the total infected population. For the CFR lower than $0.1 \%$ (Category 1), voluntary at-home isolation of infected cases is implemented. If the CFR falls in-between $0.1 \%$ and $1.0 \%$ (Categories 2 and 3), in addition to the at-home isolation, the following measures are recommended: (i) voluntary quarantine of households members of infected cases and (ii) child and adult social distancing. For the CFR exceeding 1.0\% (Categories 4 and 5), all the above measures are implemented. As effectiveness of social distancing is affected by some of the behavioral factors listed above, ${ }^{61}$ we assume a certain social distancing conformance level. Travel restrictions considered in the model included regional air and land border control for infected travelers.

\section{Optimization models}

The optimization model (budget-based or resource-based) is invoked at the beginning of every $n$th new regional outbreak epoch $(n=1,2, \ldots)$, starting from the initial outbreak region $(n=1)$. The objective of the model is to allocate some of the available mitigation resources to the new outbreak region (actual allocation) while reserving the rest of the resources for potential outbreak regions (virtual allocation). By doing so, the model seeks to progressively minimize the impact of ongoing outbreaks and the expected impact of potential outbreaks, spreading from the ongoing locations. Mitigation resources can include stockpiles of vaccine and antiviral, administration capacities for their administration, hospital beds, medical supplies, and social distancing enforcement resources, among others. The predictive mechanism of the optimization model is based on a set of regression equations obtained using single-region simulation models. In what follows, we present the construction of the optimization model and explain the solution algorithm for the overall simulation-based optimization methodology (Section 2.4).

We introduce the following general notation.

$S=$ set of all network regions,

$A^{n}=$ set of regions in which pandemic is contained at the $n$th outbreak epoch $(n=1,2, \ldots)$,

$B^{n}=$ set of ongoing regions at the $n$th outbreak epoch,

$C^{n}=$ set of unaffected regions at the $n$th outbreak epoch,

$R=$ set of available types of mitigation resources $(R=\{1,2, \ldots, r\})$,

$c_{i}=$ unit cost of type $i$ resource, $i \in R$,

$q_{i k}=$ amount of resource $i$ allocated to region $k$,

$Q_{i}^{n}=$ available amount of resource $i \in R$ at the $n$th outbreak epoch,

$M^{n}=$ budget availability at the $n$th outbreak epoch,

$H=$ set of age groups.

In both models, the optimization criterion (objective function) incorporates measures of societal and economic costs of the pandemic: the societal cost includes the cost of lost lifetime productivity of the deceased; the economic cost includes the cost of medical expenses of the recovered and deceased and the cost of lost productivity of the quarantined. To compute these costs, the following impact measures of morbidity, mortality, and quarantine are used, for each region $k$ :

$X_{h k}=$ total number of infected cases in age group $h$ who seek medical assistance,

$Y_{h k}=$ total number of infected cases in age group $h$ who do not seek medical assistance,

$D_{h k}=$ total number of deceased cases in age group $h$,

$V_{h k}=$ total number of person-days of cases in age group $h$ who comply with quarantine.

We use the following regression models, obtained using a single-region simulation of each region $k$, to estimate the above impact measures of morbidity, mortality, and social distancing (dependent variables) as a function of the independent variables (resource allocation):

$$
X_{h k}=\delta_{h k}^{0}+\sum_{i \in R} \delta_{h k}^{i} \cdot q_{i k}+\sum_{i, m \in R, i \neq m} \delta_{h k}^{i m} \cdot q_{i k} \cdot q_{m k},
$$


where $\delta^{i}$.. denotes the regression coefficient associated with resource $i$, and $\delta^{i m}$ is the regression coefficient for the interaction between resources $i$ and $m$. Similar expressions are used for $Y_{h k}, D_{h k}$, and $V_{h k}$. The quadratic form of the regression equations is adopted, as opposed to the linear, to better capture the interaction between the variables (eg, vaccine stockpile and administration capacity).

The above relationships between the impact measures and the resource allocations ought to be determined apriori of implementing a cross-regional scenario (see Section 3). Here, we consider each region $k$ as the initial outbreak region so that the values of $X_{h k}, Y_{h k}, D_{h k}$, and $V_{h k}$ are estimated for the entire pandemic period. We assume, however, that as the pandemic evolves, the disease infectivity will naturally subside. Hence, we incorporate a decay factor $\alpha^{n 66}$ to adjust the estimates of the regional impact measures at every $n$th outbreak epoch, in the following way:

$$
\begin{aligned}
& X_{h j}^{n}=\alpha^{n} X_{h j}, Y_{h j}^{n}=\alpha^{n} Y_{h j}, D_{h j}^{n}=\alpha^{n} D_{h j}, \\
& V_{h j}^{n}=\alpha^{n} V_{h j} .
\end{aligned}
$$

Alternatively, the regression equations can be re-estimated at every new outbreak epoch, for each region $k \in C^{n}$, using the single-region simulation models, where each simulation must be initialized to the current outbreak status in region $k$ in the cross-regional simulation.

In addition, we use the following regression model to estimate the probability of pandemic spread from affected region $l$ to unaffected region $k$, as a function of resources allocated to region $l$, which, in turn, impact the number of outgoing infectious travelers from the region:

$$
p_{l k}=\gamma_{l k}^{0}+\sum_{i \in R} \gamma_{l k}^{i} \cdot q_{i l}+\sum_{\substack{i, m \in R \\ i \neq m}} \gamma_{l k}^{i m} \cdot q_{i l} \cdot q_{m l},
$$

where $\gamma^{i}$ denotes the regression coefficient associated with resource $i, \gamma^{i m}$ is the regression coefficient associated with interaction between resources $i$ and $m$, and $\gamma^{0}$ represents the intercept. Consequently, the total outbreak probability for unaffected region $k$ can be found as $p_{k}=\sum_{l \in B^{n}} p_{l k}$. We use a scheme similar to Eq. 4 to progressively adjust the estimates of the regional outbreak probabilities

$$
p_{k}^{n}=\alpha^{n} p_{k}
$$

Finally, we calculate the total cost of an outbreak in region $k$ at the $n^{\text {th }}$ decision epoch as follows

$$
\begin{aligned}
& T C_{k}^{n}=\sum_{h \in H}\left(m_{h}+\bar{w}_{h}\right) X_{h k}^{n}+ \\
& \sum_{h \in H} \bar{w}_{h} \cdot Y_{h k}^{n}+\sum_{h \in H} w_{h} \cdot D_{h k}^{n}+\sum_{h \in H} w_{h} \cdot V_{h k}^{n},
\end{aligned}
$$

where

$m_{h}=$ total medical cost of an infected case in age group $h$ over his/her disease period,

$\bar{w}_{h}=$ total cost of lost wages of an infected case in age group $h$ over his/her disease period,

$\hat{w}_{h}=$ cost of lost lifetime wages of a deceased case in age group $h$,

$w_{h}=$ daily cost of lost wages of a non-infected case in age group $h$ who complies with quarantine.

\section{Quantity-based and budget-based}

\section{optimization models}

The quantity-based and budget-based optimization models have the following form.

Both models have the same objective function. The first term of the objective function represents the total cost of the new outbreak $j$, estimated at the $n$th outbreak epoch, based on

$$
\begin{aligned}
& \text { Minimize } T C_{j}^{n}\left(q_{1 j}, q_{2 j}, \ldots, q_{r j}\right)+\sum_{s \in C^{n}} T C_{s}^{n} \\
& \left(q_{1 s}, q_{2 s}, \ldots, q_{r s}\right) \cdot p_{s}^{n}
\end{aligned}
$$

\section{Quantity-based model}

subject to

$$
\begin{aligned}
& q_{i j}+\sum_{s \in C^{n}} q_{i s} \cdot p_{s}^{n} \leq Q_{i}^{n} \quad \forall i \in R, \\
& q_{i j}, q_{i s} \geq 0 \quad \forall i \in R .
\end{aligned}
$$

\section{Budget-based model}

subject to

$$
\begin{aligned}
& \sum_{i \in R} c_{i} \cdot q_{i j}+\sum_{s \in C^{n}} \sum_{i \in R} c_{i} \cdot q_{i s} \cdot p_{s}^{n} \leq M^{n}, \\
& q_{i j}, q_{i s} \geq 0 \quad \forall i \in R .
\end{aligned}
$$

the actual resource allocation $\left\{q_{1 j}, q_{2 j}, \ldots q_{r j}\right\}$ (see Eq. 6). The second term represents the total expected cost of outbreaks in currently unaffected regions, based on the virtual allocations $\left\{q_{1 s}, q_{2 s}, \ldots, q_{r s}\right\}$ and the regional outbreak probabilities $p^{n}{ }_{s}$ (Eq. 5).

In the quantity-based model, the set of constraints assures that for each resource $i$, the total quantity allocated (current and virtual, both nonnegative) does not exceed the total resource availability at the $n$th decision epoch. 
Both the objective function and the availability constraints are nonlinear in the decision variables.

In the budget-based model, the first constraint relates the total available budget at $n$th decision epoch with the total cost of the resources to be allocated (current and virtual). The second constraint assures a nonnegative allocation.

\section{Solution algorithm}

The solution algorithm for our dynamic predictive simulation optimization methodology is given below.

1. Estimate regression equations for each region using the single-region simulation model.

2. Begin the cross-regional simulation model.

3. Initialize the sets of regions: $A^{n}=\phi, B^{n}=\phi, C^{n}=S$

4. Select randomly the initial outbreak region $j$. Set $n=1$.

5. Update sets of regions: $B^{n} \leftarrow B^{n} \cup\{j\}$ and $C^{n} \leftarrow$ $C^{n} \backslash\{j\}$.

6. Solve the resource allocation model (budget or quantity) for region $j$. Update the total resource availabilities.

7. If $B^{n} \neq \phi$, do step 8. Else, do step 10 .

8. (a) For each ongoing region, implement a next day run of its single-region simulation.

(b) Check the containment status of each ongoing region. Update sets $A^{n}$ and $B^{n}$, if needed.

(c) For each unaffected region, calculate its outbreak probability.

(d) Based on the outbreak probability values, determine if there is a new outbreak region(s) $j$.

If there is no new outbreak(s), go to step 7. Otherwise, go to step 9.

9. For each new outbreak region $j$,

(a) Increment $n \leftarrow n+1$.

(b) Update sets $B^{n} \leftarrow B^{n} \cup\{j\}$ and $C^{n} \leftarrow C^{n} \backslash\{j\}$.

(c) Re-estimate regression equations for each region $k \in B^{n} \cup C^{n}$ using the single-region simulations, where each simulation is initialized to the current outbreak status in the region (optional step; alternatively, use Eq. 4 and Eq. 5).

(d) Solve the resource allocation model (budget or quantity) for region $j$.

(e) Update the total resource availabilities.

10. Calculate the total cost for each contained region and update the overall pandemic cost.

\section{Testbed}

A testbed scenario included a network of four counties in

Florida: Hillsborough, Miami Dade, Duval, and Leon, with population of 1.0, 2.2, 0.8, and 0.25 million people, respectively. The H5N1 virus was considered. A basic unit of time $\Delta t$ for population and disease dynamics models was taken as one hour. Regional simulations were run for a period (up to 180 days) until the daily infection rate approached near zero (see Section 3). Below we present the details on selecting parameter values for our models. Most of the testbed data can be found in the supplement. ${ }^{67}$

\section{Parameter values for population and disease dynamics models}

Demographic and social dynamics data for each region were extracted from the U.S. Census ${ }^{68}$ and the National Household Travel Survey. ${ }^{69}$ Daily (hourly) schedules were adopted from Das and Savachkin, 2008. ${ }^{43}$

Each infected person was assigned a daily travel probability of $0.24 \%,{ }^{69}$ of which $7 \%$ was by air and $93 \%$ by land. The probabilities of travel among the four regions were calculated using traffic volume data (Table 1). ${ }^{70-73}$ Infection detection probabilities for border control for symptomatic cases were assumed to be $95 \%$ and $90 \%$, for air and land, respectively. These values were reduced by $70 \%$ for asymptomatic cases. The value of $\omega$ in Eq. 1 was taken as 0.01 . The values of regional outbreak probabilities $P_{t}$ were calculated once at the end of every day.

The instantaneous force of infection applied to contact $i$ at time $t$ (Eq. 2) was modeled as

$$
\lambda_{i}(t)=-\ln \left(1-p_{i}(t)\right), \text { where } p_{i}(t)=\alpha_{i}-\delta \theta_{i}(t)
$$

where $\alpha_{i}$ is the age-dependent base instantaneous infection probability of contact $i, \theta_{i}(\mathrm{t})$, is her status of vaccination at time $t$ ( 0 or 1$)$, and $\delta$ is the vaccine efficacy, measured as the reduction in the base instantaneous infection probability (achieved after 10 days). ${ }^{50}$

The values of age-dependent base instantaneous infection probabilities were adopted from Germann, $2006^{37}$ (see Table 2). The disease natural history for H5N1

Table I Inter-regional travel probabilities

\begin{tabular}{lllll}
\hline Origin/destination & \multicolumn{4}{l}{ Inter-regional travel probability } \\
\cline { 2 - 5 } & Hillsborough & Miami D & Duval & Leon \\
\hline Hillsborough & 0.00 & 0.60 & 0.27 & 0.13 \\
Miami D & 0.74 & 0.00 & 0.16 & 0.10 \\
Duval & 0.61 & 0.29 & 0.00 & 0.10 \\
Leon & 0.52 & 0.31 & 0.17 & 0.00 \\
\hline
\end{tabular}


Table 2 Instantaneous infection probabilities

\begin{tabular}{llllll}
\hline Age group & $\mathbf{0 - 5}$ & $\mathbf{6 - 1 9}$ & $\mathbf{2 0 - 2 9}$ & $\mathbf{3 1 - 6 5}$ & $\mathbf{6 6 - 9 9}$ \\
\hline$\alpha_{i}$ & 0.156 & 0.106 & 0.205 & 0.195 & 0.344 \\
\hline
\end{tabular}

virus included a latent period of 29 hours, an incubation period of 46 hours, and an infectiousness period between 29 and 127 hours. $^{74}$

Base mortality probabilities ( $\mu_{i}$ in Eq. 3 ) were determined using the statistics recommended by the Working Group on Pandemic Preparedness and Influenza Response. ${ }^{44}$ This data shows the percentage of mortality for age-based high-risk cases (HRC) (Table 3, columns 1-3). Mortality probabilities (column 4) were estimated under the assumption that highrisk cases are expected to account for of the total number of fatalities, for each age group. ${ }^{44}$

\section{Calibration of the single-region models}

Single-region simulation models were calibrated using the basic reproduction number $\left(R_{0}\right)$ and the infection attack rate (IAR). $R_{0}$ is defined as the average number of secondary infections produced by a typical infected case in a totally susceptible population. IAR is defined as the ratio of the total number of infections over the pandemic period to the size of the initial susceptible population. To determine $R_{0}$, all infected cases inside the simulation were classified by generation of infection. ${ }^{25,34}$ The value of $R_{0}$ was calculated as the average reproduction number of a typical generation in the early stage of the pandemic, with no interventions implemented (baseline scenario). ${ }^{25}$ Historically, $R_{0}$ values for PI ranged between 1.4 and 3.9..$^{29,34}$ To attain similar values, we calibrated the hourly contact rates of mixing groups (original rates were adopted from). ${ }^{37}$ For the four regions, the average value of $R_{0}$ was 2.54 , which represented a high transmissibility scenario. The values of regional IAR averaged 0.538 .

\section{Mitigation related parameters}

Mitigation resources included stocks of vaccines and antiviral, capacities for vaccination and antiviral application, and quarantine enforcement resources (required to achieve a

Table 3 Mortality probabilities for different age groups

\begin{tabular}{llll}
\hline Age group & \% High-risk cases & $\begin{array}{l}\text { \% Mortality in } \\
\text { high-risk cases }\end{array}$ & $\mu_{i}$ \\
\hline $0-19$ & 6.4 & 9.0 & 0.007 \\
$20-64$ & 14.4 & 40.9 & 0.069 \\
$65+$ & 40.0 & 34.4 & 0.162 \\
\hline
\end{tabular}

target conformance level). We assumed a 24-hour deployment delay following detection of the first infection case. ${ }^{49}$

The vaccination risk group included individuals younger than 5 years and older than 65 years. ${ }^{75}$ The risk group for antiviral included individuals below 15 years and above 55 years. $^{75,76}$ The efficacy levels for the vaccine ( $\delta$ in Eq. 7) and antiviral ( $\tau$ in Eq. 3 ) were assumed to be $40 \%{ }^{35,77}$ and $30 \%$, respectively. We assumed a $95 \%$ target population conformance for both measures. The immunity development period for the vaccine was taken as 10 days. ${ }^{50}$

A version of the CDC guidance for quarantine and isolation for Category 5 was implemented (see Section 4). Once the CFR has reached $1.0 \%$, the following policy was declared and remained in effect for 14 days: ${ }^{42}$ (i) individuals below a prespecified age $\xi$ (22 years) stayed at home during the entire policy duration; (ii) of the remaining population, a certain proportion $\phi$ stayed at home and was allowed a one-hour leave, every three days, to buy essential supplies; ${ }^{78}$ (iii) the remaining (1- $\phi)$ non-compliant proportion followed a regular schedule. All testbed scenarios considered the quarantine conformance level $\phi$ (a decision variable) bounded between $50 \%$ and $80 \%{ }^{61}$

An outbreak was considered contained if the daily infection rate did not exceed five cases, for seven consecutive days. Once contained, a region was simulated for an additional 10 days for accurate estimation of the pandemic statistics. A $2^{5}$ statistical design of experiment was used to estimate the regression coefficient values of the significant decision factors and their interactions (Section 2.3).

The simulation code was developed using $\mathrm{C}++$. The running time for a cross-regional simulation replicate averaged 19 minutes on a Pentium $3.40 \mathrm{GHz}$ PC with $4.0 \mathrm{~GB}$ of RAM.

\section{Comparison of two allocation models: sensitivity analysis}

This section presents a performance comparison of the quantity-based and budget-based allocation models. We also present a sensitivity analysis for assessing the marginal impact of changes in the total resource availability. The marginal impact is measured by the change in the total pandemic cost (averaged over multiple replicates), resulting from a unit change in the total quantity/budget availability.

Table 4 summarizes vaccination and antiviral treatment resource requirements for each region, based on the composition of the risk groups (see Section 3). The table also shows the resource costs, ${ }^{79-81}$ budget requirement by each resource, and the total required budget. 
Table 4 Regional resource and budget requirements

\begin{tabular}{|c|c|c|c|c|c|c|c|}
\hline \multirow{2}{*}{$\begin{array}{l}\text { Region } \\
\text { (population) }\end{array}$} & \multicolumn{5}{|c|}{ Resource requirements by region } & \multirow{2}{*}{$\begin{array}{l}\text { Cost of } \\
\text { resource }\end{array}$} & \multirow{2}{*}{$\begin{array}{l}\text { Required } \\
\text { budget }\end{array}$} \\
\hline & $\begin{array}{l}\text { Hillsb } \\
(1,007,916)\end{array}$ & $\begin{array}{l}\text { Miami D } \\
(2,209,702)\end{array}$ & $\begin{array}{l}\text { Duval } \\
(852,168)\end{array}$ & $\begin{array}{l}\text { Leon } \\
(248,761)\end{array}$ & $\begin{array}{l}\text { Total } \\
(4,318,547)\end{array}$ & & \\
\hline \multicolumn{8}{|l|}{ Resource } \\
\hline Vaccine stock & 305,036 & 679,181 & $24 I, 522$ & 76,007 & $\mathrm{I}, 30 \mathrm{I}, 745$ & $\$ 8.48 /$ dose & $\$ 11.038 \mathrm{M}$ \\
\hline Antiviral stock & 415,294 & 749,058 & 460,393 & 105,307 & $1,730,052$ & $\$ 60 /$ dose & $\$ 103.803 \mathrm{M}$ \\
\hline No. antiv. nurses & 650 & $\mathrm{I}, 104$ & 786 & 166 & 2,706 & $\$ 27 / \mathrm{hr} 8 \mathrm{hr} /$ day, 50 days & $\$ 29.226 \mathrm{M}$ \\
\hline \multirow[t]{2}{*}{ No. vacc. nurses } & 1,059 & 2,358 & 839 & 264 & 4,520 & $\$ 27 / \mathrm{hr} 8 \mathrm{hr} /$ day, I4 days & $\$ 13.668 \mathrm{M}$ \\
\hline & & & & & \multicolumn{2}{|c|}{ Total budget requirement } & $\$ 157.737 \mathrm{M}$ \\
\hline
\end{tabular}

Table 5 shows the costs of lost productivity and medical expenses adopted from Meltzer, $1999^{44}$ and adjusted for inflation for the year of $2010 .^{82}$

Comparison of the two allocation models was done at different levels of the total resource availability relative to the total requirement. The total resource quantity requirements are shown in Table 4 along with the corresponding required budget. The sensitivity analysis was performed for quantity levels of $80 \%, 65 \%, 50 \%, 35 \%$, and $20 \%$ of the total quantity requirement, for each resource. The corresponding budget values were computed using resource unit costs from Table 4.

Figures $3 \mathrm{a}$ and $3 \mathrm{~b}$ below show the dynamics of the average number of infected cases and the average number of fatalities for different levels of resource availability. Figure 4 shows the corresponding dynamics for the average total pandemic cost. For illustrative purposes, we also show the average number of regional outbreaks, for both allocation models, in the testbed scenario involving four regions, with Hillsborough as the initial outbreak region (Table 6).

Table 5 Values of pandemic impact measures (societal and economic costs)

\begin{tabular}{|c|c|}
\hline $\begin{array}{l}\text { Pandemic impact measure } \\
\text { (age group, years) }\end{array}$ & Value US\$ \\
\hline $\begin{array}{l}\text { Average cost of lost lifetime productivity } \\
\text { of a deceased case }(0-19)\end{array}$ & $\$ 1,336,347.86$ \\
\hline $\begin{array}{l}\text { Average cost of lost lifetime productivity } \\
\text { of a deceased case }(20-64)\end{array}$ & $\$ 1,370,987.28$ \\
\hline $\begin{array}{l}\text { Average cost of lost lifetime productivity } \\
\text { of a deceased case (65-99) }\end{array}$ & $\$ 98,959.24$ \\
\hline $\begin{array}{l}\text { Average cost of lost productivity and medical } \\
\text { expenses of a recovered/deceased case }(0-19)\end{array}$ & $\$ 5,078.48$ \\
\hline $\begin{array}{l}\text { Average cost of lost productivity and medical } \\
\text { expenses of a recovered/deceased case (20-64) }\end{array}$ & $\$ 10,466.68$ \\
\hline $\begin{array}{l}\text { Average cost of lost productivity and medical } \\
\text { expenses of a recovered/deceased case (65-99) }\end{array}$ & $\$ 11,566.09$ \\
\hline $\begin{array}{l}\text { Average daily cost of lost productivity } \\
\text { of a non-infected quarantined case (20-99) }\end{array}$ & $\$ 432.54$ \\
\hline
\end{tabular}

As expected, the curves for all impact measures (average total number of infected, deaths, and total cost) exhibit a downward trend, for both allocation models, as the total resource availability increases. Increased total resource availability not only mediates a regional pandemic impact but also reduces the probability of spread to unaffected regions. For both models, as the resource availability approaches the resource requirement (starting from approximately 60\%), the impact curves show a converging behavior, whereby the marginal utility of additional resource availability diminishes. This can be explained by noting that the total resource requirement was determined assuming the worst case scenario when all regions would be affected and provided with adequate resources to cover their respective populations at risk. It can also be observed that for all three measures, the budget-based model dominates the quantity-based model at all levels of resource availability. This can be attributed to the fact that the budget-based model, being less restrictive as having only one constraint (Section 1), also takes advantage of its ability to balance the relative cost and effectiveness of individual resources, such as vaccine, antiviral, etc. The difference in the performance of two models was as high as 339,079 infected cases, 19,724 fatalities, and 17.84 $\mathrm{M}$ in the pandemic cost (all attained at the $20 \%$ resource availability). The difference in policy performance gradually diminishes as the resource availability increases and as available quantities become close to be sufficient to cover the entire populations at risk in all regions: at $80 \%$ availability, the gap was 118,124 infected, 4,318 fatalities, and 5.07M in the pandemic cost.

\section{Conclusions}

Effective mitigation of influenza pandemics has to invariably rely on understanding of evolution of disease and population dynamics and intelligent resource allocation. As recently pointed by the IOM, the existing models fall short of providing dynamic decision support, which incorporates broader outcome measures including "costs and benefits 


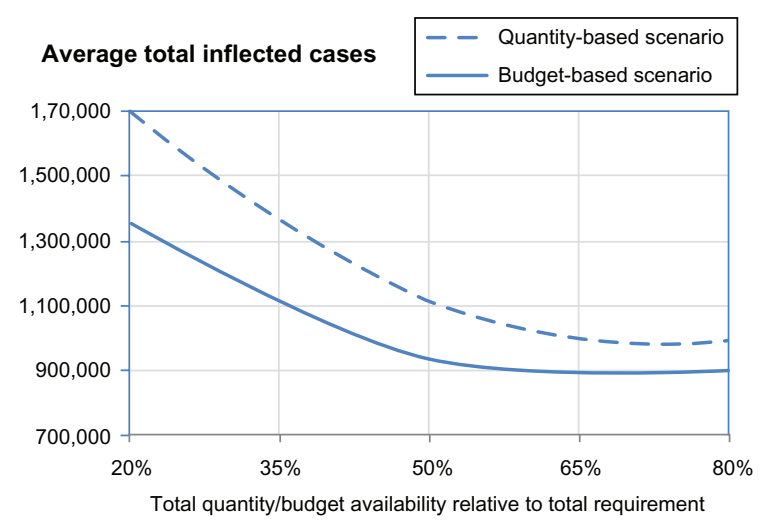

(a)

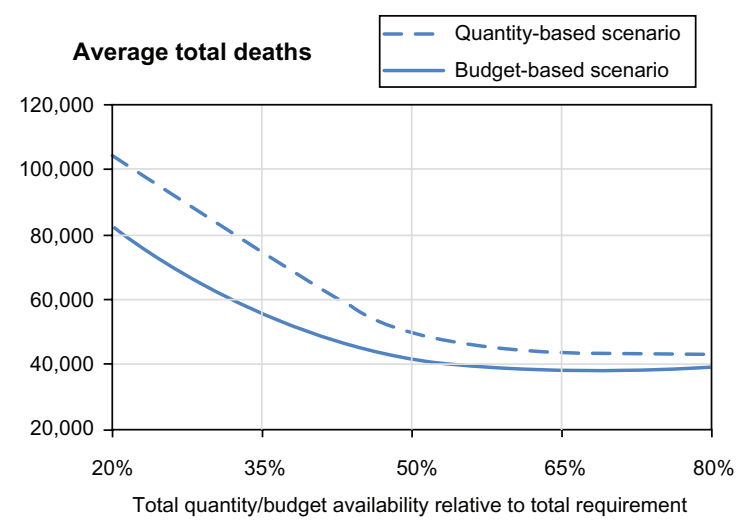

(b)

Figure 3 Sensitivity analysis on total resource availability (measured in terms of the average number of infected (a) and the average number of deaths (b)).

of intervention". In this paper, we present two large-scale simulation-based optimization models specifically aimed at addressing these challenges.

The models support development of dynamic predictive resource allocation strategies for a network of regional outbreaks. In both models, the underlying simulation mimics the disease and population dynamics of the affected regions. The quantity-based optimization model generates a progressive allocation of resource quantities, whereas the budget-based model aims at allocating the total resource budget. Both models seek to minimize the impact of ongoing and expected outbreaks. The impact measures incorporate morbidity, mortality, and social distancing, translated into the societal and economic costs of lost productivity and medical expenses. The models were calibrated using historic pandemic data and implemented on a sample cross-regional outbreak in Florida, with over 4M inhabitants.

Summary of the main results. We observed that for both models, the marginal utility of additional resource availability

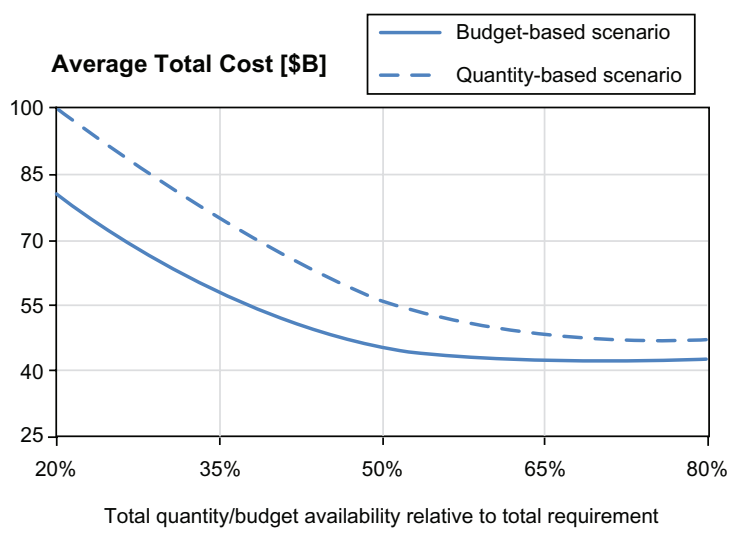

Figure 4 Sensitivity analysis on total resource availability (measured in terms of the total cost). was steadily diminishing as the resource availability approached the resource requirement for a worst case scenario, in which all regions would be affected. We also showed that the quantity-based model was inferior to the budgetbased model at different levels of resource availability. While the budget-based model includes the quantity-based solution as a special case, the former takes advantage of its inherent ability to balance the different relative cost and effectiveness of individual resources. Moreover, the budget-based model is less restrictive (the only constraint is the total available budget), and therefore, it has a richer geometry of feasible solutions at any decision epoch of pandemic evolution. The difference in model performance was particularly noticeable at lower levels of resource availability, which is in accordance with a higher marginal utility of additional availability at that levels. Hence, the budget-based model can be most useful in scenarios with very limited supply of resources.

Contributions of the model. The methodology presented in this paper is one of the first attempts to offer dynamic predictive decision support for pandemic mitigation, which incorporates measures of societal and economic impact. Our comparison study of the budget-based versus quantity-based progressive cross-regional resource allocations is also novel. In addition, our simulation model represents one of the first of its kind in considering a broader range of social behavioral aspects, including vaccination and antiviral treatment conformance. The simulation features a flexible design which can be particularized to a broader range of pharmaceutical and non-pharmaceutical interventions and more granular mixing groups.

Based on our methodology, we have also developed a decision-aid simulator with a GUI which is made freely available to general public through our web site at http:// imse.eng.usf.edu/pandemics.aspx. The simulator allows the 
Table 6 Average number of regional outbreaks for both models

\begin{tabular}{llll}
\hline & \multicolumn{3}{l}{ Total resource availability } \\
\cline { 2 - 4 } Model & $\mathbf{2 0 \%}$ & $\mathbf{5 0 \%}$ & $\mathbf{8 0 \%}$ \\
\hline Quantity-based & 1.89 & 1.44 & 1.38 \\
Budget-based & 1.63 & 1.33 & 1.20 \\
\hline
\end{tabular}

input of data for regional demographic and social dynamics, and disease related parameters. It is intended to assist public health decision makers in conducting customized what-if analysis for assessment of mitigation options and development of policy guidelines. Examples of such guidelines include targeted risk groups for vaccination and antiviral treatment, social distancing policies (eg, thresholds for declaration and lifting, closure options (ie, household-based, schools, etc.), and compliance targets), and guidelines for travel restrictions.

Limitations of the model. Lack of reliable data prevented us from considering geo-spatial aspects of mixing group formation in the testbed implementation. We also did not consider the impact of public education and use of personal protective measures (eg, face masks) on transmission, again due to a lack of effectiveness data. ${ }^{83}$ We did not study the marginal effectiveness of individual resources due to a considerable uncertainty about the transmissibility of a future pandemic virus and efficacy of vaccine and antiviral. For the same reason, the vaccine and antiviral risk groups considered in the testbed can be adjusted, as different prioritization schemes have been suggested in the literature, based on predicted characteristics of a future virus. The form of social distancing implemented in the testbed can also be modified as a variety of schemes can be found in the literature, including geographical and social targeting. Effectiveness of these approaches is substantially influenced by the policy compliance, for which limited accurate data support exists. It will thus be vital to gather the most detailed data on the epidemiology of a new virus and the population dynamics early in the evolution of a pandemic, and to expeditiously analyze it to adjust the interventions accordingly.

\section{Disclosure}

The authors disclose no conflicts of interest.

\section{References}

1. Longini IM, Halloran ME, Nizam A, Yang Y. Containing Pandemic In uenza with Antiviral Agents. Am J Epidemiol. 2004;159: 623-633.

2. Centers for Disease Control and Prevention (CDC) . Avian in uenza: Current H5N1 situation. http://www.cdc.gov/flu/avian/outbreaks/current. $\mathrm{htm} \#$ clusters 2008. Last accessed on 02/09/2008.
3. World Health Organization (WHO). Cumulative number of confirmed human cases of Avian In uenza A(H5N1) reported to WHO. http://www. who.int/csr/disease/avian_influenza/country/cases_table_2010_03_04/ en/index.html 2010. Last accessed on 03/04/2010.

4. World Health Organization. Pandemic (H1N1) 2010 - update 90. http:// www.who. int/csr/don/2010_03_05/en/index.html 2010. Last accessed on $03 / 04 / 2010$.

5. Arthur Schoenstadt. Spanish Flu. http://flu.emedtv.com/spanish-flu/ spanish-flu.html 2009. Last accessed on 12/15/2009.

6. Fedson DS, Hant S. Pandemic in uenza and the global vaccine supply. Clin Infect Dis. 2003;36:1552-1561.

7. Aunins JG, Lee AL, Volkin DB. Vaccine production. Boca Raton: CRC Press LLC2 ed; 2000.

8. World Health Organization. Pandemic (H1N1) 2009 vaccine deployment update -17 December 2009. http://www.who.int/csr/disease/swineflu/ vaccines/h1n1_vaccination_deployment_update_20091217.pdf 2009.

9. WHO Global Influenza Programme. Pandemic In uenza Preparedness and Response tech. rep.World Health Organization 2009.

10. Centers for Disease Control and Prevention (CDC). Preparing for Pandemic Influenza. http://www.cdc.gov/flu/pandemic/preparedness. $\mathrm{htm}$ 2007. Last accessed on 04/27/2009.

11. US Department of Health \& Human Services. HHS pandemic in uenza plan. http://www.hhs.gov/pandemicflu/plan/ 2007. Last accessed on 03/27/2009.

12. Handel A, Longini I, Antia R. Towards a quantitative understanding of the within-host dynamics of in uenza A infections. Epidemics. 2009;1:185-195.

13. Pourbohloul B, Ahued A, Davoudi B, et al. Initial human transmission dynamics of the pandemic (H1N1) 2009 virus in North America. Influenza Other Respi Viruses. 2009;3:215-222.

14. Atkinson M, Wein L. Quantifying the Routes of Transmission for Pandemic Influenza. Bull Math Biol. 2008;70: 820-867.

15. Pitzer VE, Olsen SJ, Bergstrom CT, Dowell SF, Lipsitch M. Little evidence for genetic susceptibility to in uenza A (H5N1) from family clustering data. Emerg Infect Dis. 2007;13:1074-1076.

16. Pitzer VE, Leung GM, Lipsitch M. Estimating variability in the transmission of severe acute respiratory syndrome to household contacts in Hong Kong, China. Am J Epidemiol. 2007;166:355-359.

17. Yang Y, Sugimoto JD, Halloran ME, et al. The transmissibility and control of pandemic in uenza A (H1N1) virus. Science. 2009;326: 729-733.

18. Yang Y, Halloran ME, Sugimoto J, Longini Jr IM. Detecting humanto-human transmission of avian in uenza A (H5N1). Emerg Infect Dis. 2007;13:1348-1353.

19. Cauchemez S, Carrat F, Viboud C, Valleron A, Boelle P. A Bayesian (MCMC) approach to study transmission of in uenza: application to household longitudinal data. Statist Med. 2004;23:3469-3487.

20. Colizza V, Barrat A, Barthelemy M, Vespignani A. The role of the airline transportation network in the prediction and predictability of global epidemics. Proc Natl Acad Sci U S A. 2006;103:2015-2020.

21. Epstein JM, Parker J, Cummings D, Hammond RA. Coupled contagion dynamics of fear and disease: mathematical and computational explorations. PLoS One. 2008;3.

22. Epstein JM, Goedecke DM, Yu F, Morris RJ, Wagener DK, Bobashev GV. Controlling pandemic flu: the value of international air travel restrictions. PLoS One. 2007;2.

23. Halloran ME. Invited commentary: Challenges of using contact data to understand acute respiratory disease transmission. Am J Epidemiol. 2006;164:945.

24. Scharfstein DO, Halloran ME, Chu H, Daniels MJ. On estimation of vaccine efficacy using validation samples with selection bias. Biostatistics. 2006;7:615.

25. Glass R, Beyeler W, Min H. Targeted social distancing design for pandemic influenza. Emerg Infect Dis. 2006;12:1671-1681.

26. Lipsitch M, Cohen T, Murray M, Levin BR. Antiviral resistance and the control of pandemic influenza. PLoS Medicine. 2007;4: $111-115$. 
27. Nigmatulina Karima R, Larson Richard C. Living with influenza: Impacts of government imposed and voluntarily selected interventions. Eur J Oper Res. 2009;195:613-627.

28. Wu JT, Riley S, Fraser C, Leung G. Reducing the impact of the Next influenza pandemic using household-Based public health interventions. PLoS Med. 2006;3:1532-1540.

29. Mills C, Robins J, Lipsitch M. Transmissibility of 1918 pandemic influenza. Nature. 2004;432:904-906.

30. Patel R, Longini I, Halloran M. Finding optimal vaccination strategies for pandemic in uenza using genetic algorithms. $J$ Theor Biol. 2005;234:201-212.

31. Colizza V, Barrat A, Barthelemy M, Valleron A, Vespignani A. Modeling the worldwide spread of pandemic influenza: baseline case and containment interventions. PLOS Medicine. 2007;4:95-110.

32. Cooley P, Ganapathi L, Ghneim G, Holmberg S, Wheaton W, Hollingsworth C. Using influenza-like illness data to reconstruct an influenza outbreak. Math Comput Model. 2008:929-939.

33. National Institute of General Medical Sciences . Models of Infectious Disease Agent Study. http://www.nigms.nih.gov/Initiatives/MIDAS/ 2008. Last accessed on 09/10/2008.

34. Ferguson NM, Cummings D, Cauchemez S, et al. Strategies for containing an emerging influenza pandemic in Southeast Asia. Nature. 2005;437:209-214.

35. Longini IM, Nizam A, Shufu X, et al. Containing Pandemic Influenza at the Source. Science. 2005;309:1083-1087.

36. Ferguson N, Cummings DA, Fraser C, Cajka J, Philip Cooley C, Burke DS. Strategies for mitigating an influenza pandemic. Nature. 2006;442:448-452.

37. Germann T, Kadau K, Longini IM, Macken C. Mitigation strategies for pandemic influenza in the United States. Proc Natl Acad Sci USA. 2006;103: 5935-5940.

38. Eubank S, Guclu H, Kumar V, et al. Modelling disease outbreaks in realistic urban social networks. Nature. 2004;429:180-184.

39. Halloran ME, Ferguson NM, Eubank S, Longini I. Modeling targeted layered containment of an influenza pandemic in the United States. Proc Natl Acad Sci U S A. 2008;105:4639-4644.

40. MIDAS. Report from the Models of Infectious Disease Agent Study (MIDAS) Steering Committee http://www.nigms.nih.gov/ News/Reports/midas_steering_050404.htm 2004. Last accessed on 09/10/2008.

41. Committee on Modeling Community Containment for Pandemic Influenza. Modeling community containment for pandemic influenza: A letter report. http://www.nap. edu/catalog/11800.html 2006. Last accessed on 12/09/2008.

42. Centers for Disease Control and Prevention (CDC). Interim prepandemic planning guidance: Community strategy for pandemic influenza mitigation in the United States. http://www.pandemicflu. gov/plan/community/community_mitigation.pdf 2007. Last accessed on $04 / 01 / 2009$.

43. Das T, Savachkin A. A large scale simulation model for assessment of societal risk and development of dynamic mitigation strategies. IIE Transactions. 2008;40:893-905.

44. Meltzer M, Cox N, Fukuda K. The economic impact of pandemic influenza in the United States: Priorities for intervention. Emerg Infect Dis. 1999;5:659-671.

45. Wu JT, Riley S, Leung G. Spatial considerations for the allocation of pre-pandemic influenza vaccination in the United States. Proc Biol Sci. 2007;274:2811-2817.

46. Lawless JF, Lawless JF. Statistical models and methods for lifetime data. New York: Wiley; 1982.

47. Diekmann O, Heesterbeek JAP. Mathematical epidemiology of infectious diseases: Model building, analysis and interpretation. New York: Wiley; 2000.

48. Brundage JF, Shanks GD. Deaths from bacterial pneumonia during 1918-19 influenza pandemic. Emerg Infect Dis. 2008;14:1193.
49. Centers for Disease Control and Prevention (CDC). CDC influenza operational plan. http://www.cdc.gov/flu/pandemic/cdcplan.htm 2006. Last accessed on 03/27/2009.

50. Pasteur S. Influenza A(H1N1) 2009 Monovalent Vaccine. http://www. fda.gov/downloads/biologicsbloodvaccines/vaccines/approvedproducts/ ucm182404. pfd 2009. Last accessed on 11/18/2009.

51. Blendon RJ, Koonin LM, Benson JM, et al. Public response to community mitigation measures for pandemic influenza. Emerg Infect Dis. 2008;14:778.

52. Sadique MZ, Edmunds WJ, Smith RD, et al. Precautionary behavior in response to perceived threat of pandemic influenza. Emerg Infect Dis. 2007;13:1307.

53. Maunder R, Hunter J, Vincent L, et al. The immediate psychological and occupational impact of the 2003 SARS outbreak in a teaching hospital. Can Med Assoc J. 2003;168:1245-1251.

54. Robertson E, Hershenfield K, Grace SL, Stewart DE. The psychosocial effects of being quarantined following exposure to SARS: a qualitative study of Toronto health care workers. Can J Psychiatry. 2004;49:403-407.

55. Pearson ML, Bridges CB, Harper SA. Influenza vaccination of healthcare personnel Recommendations of the Healthcare Infection Control Practices Advisory Committee (HICPAC) and the Advisory Committee on Immunization Practices (ACIP) [Internet]. 2006. Last accessed on 10/28/2009

56. Keane M, Walter M, Patel B, et al. Confidence in vaccination: a parent model. Vaccine. 2005;23:2486-2493.

57. Niederhauser VP, Baruffi G, Heck R. Parental decision-making for the varicella vaccine. J Pediatr Health Care. 2001;15: 236-243.

58. Rhodes SD, Hergenrather KC. Exploring hepatitis B vaccination acceptance among young men who have sex with men: facilitators and barriers. Prev Med. 2002;35:128-134.

59. Rosenthal SL, Kottenhahn RK, Biro FM, Succop PA. Hepatitis B vaccine acceptance among adolescents and their parents. J Adolesc Health. 1995; 17:248-255.

60. Smailbegovic M, Laing G, Bedford H. Why do parents decide against immunization? The effect of health beliefs and health professionals Child Care Health Dev. 2003;29:303.

61. Colardo Department of Human Services Division of Mental Health. Pandemic influenza: Quarantine, isolation and social distancing. http:// www.flu.gov/news/ colorado_toolbox.pdf 2009. Last accessed on 10/28/2009.

62. Safranek TJ, Lawrence DN, Kuriand LT, et al. Reassessment of the association between Guillain-Barré syndrome and receipt of swine influenza vaccine in 1976-1977: results of a two-state study. $J$ Epidemiol. 1991;133:940.

63. Cummings KM, Jette AM, Brock BM, Haefner DP. Psychosocial determinants of immunization behavior in a swine influenza campaign. Med Care. 1979;17:639-649.

64. The New Yorker. The fear factor. http://www.newyorker.com/talk/ comment/2009/ 10/12/091012taco_talk_specter 2009. Last accessed on $10 / 28 / 2009$.

65. The New York Times. Doctors swamped by swine flu vaccine fears. http:// www.msnbc.msn.com/id/33179695/ns/health-swine_flu/ 2009. Last accessed on 10/28/2009.

66. Gosavi A, Das TK, Sarkar S. A simulation based learning automata framework for solving semi-Markov decision problems under long run average reward. IIE Transactions in Operations Engineering. 2001;36:557-567.

67. Savachkin A, Uribe-Sánchez A, Das T, Prieto D, Santana A, Martinez D. Supplemental data and model parameter values for cross-regional simulation-based optimization testbed. http://imse.eng.usf.edu/ pandemic/supplement.pdf 2010. Last accessed on 04/15/2010.

68. U.S Census Bureau. 2001 American community survey. http://www. census.gov/ prod/2001 pubs/statab/sec01.pdf 2000. Last accessed on 03/27/2009. 
69. Bureau of transportation statistics. 2001 National household travel survey (NTHS). http://www.bts.gov/programs/national_household_ travel_survey/2002. Last accessed on 12/09/2008.

70. Tampa International Airport. Daily Schedule http://www.tampaairport. com/ 2008. Last accessed on 12/09/2008.

71. Miami International Airport. http://www.miami-airport.com/. Last accessed on 12/09/2008.

72. Jacksonville Aviation Authority. Daily Schedule. http://www.jaa.aero/ General/ Default.aspx 2008. Last accessed on 12/09/2008.

73. Tallahassee Regional Airport. Daily Schedule. http://www.talgov.com/ airport/ index.cfm 2008. Last accessed on 12/09/2008.

74. Writing committee of the World Health Organization (WHO). Consultation on human influenza $\mathrm{A} / \mathrm{H} 5$. Avian influenza $\mathrm{A}(\mathrm{H} 5 \mathrm{~N} 1)$ infection in humans. N Engl J Med. 2005;353:1374-1385.

75 World Health Organization (WHO). WHO guidelines on the use of vaccine and antivirals during influenza pandemics. http://www.who.int/ csr/resources/publications/influenza/11_29_01_A.pdf 2004. Retreived 03/27/2009.

76. Institute of Medicine (IOM). Antivirals for pandemic influenza Guidance on developing a distribution and dispensing. program. The National Academies Press. 2008.
Academies Press.. 2008.

77. Treanor J, Campbell J, Zangwill K, Rowe T, Wolff M. Safety and immunogenicity of an inactivated subvirion influenza $\mathrm{A}(\mathrm{H} 5 \mathrm{~N} 1)$ vaccine. N Engl J Med. 2006;3554:1343.

78. Blendon RJ, DesRoches CM, Cetron MS, Benson JM, Meinhardt T, Pollard W. Attitudes toward the use of quarantine in a public health emergency in four countries. Health Aff. 2006;25:15-25.

79. PayScale. Job: Registered Nurse (RN). http://www.payscale.com/ rcsearch.aspx? country=US\&str=Registered+Nurse + . Last accessed on $12 / 28 / 2009$.

80. Centers for Disease Control and Prevention. CDC vaccine price 1list. http://www. cdc.gov/vaccines/programs/vfc/cdc-vac-price-list.htm\#flu. Last accessed on 12/28/2009.

81. PharmacyChecker.com. Pricing \& ordering comparisons. http://www. pharmacychecker.com/Pricing.asp?DrugId=36260\&DrugStrengthId= $61300 \&$ sortby=Price. Last accessed on 12/28/2009.

82. Halfhill T. Inflation Calculator. http://www.halfhill.com/inflatation.html 2009. Last accessed on 12/04/2009.

83. Bell DM, et al. Non-pharmaceutical interventions for pandemic influenza, national and community measures. Emerg Infect Dis. 2006; $12: 88$
Journal of Multidisciplinary Healthcare

\section{Publish your work in this journal}

The Journal of Multidisciplinary Healthcare is an international, peerreviewed open-access journal that aims to represent and publish research in healthcare areas delivered by practitioners of different disciplines. This includes studies and reviews conducted by multidisciplinary teams as well as research which evaluates the results or conduct of such teams or

\section{Dovepress}

healthcare processes in general. The journal covers a wide range of areas and welcomes submission from practitioners at all levels, from all over the world. The manuscript management system is completely online and includes a very quick and fair peer-review system. Visit http://www.dovepress.com/testimonials.php to read real quotes from published authors. 